\title{
Nitroxide Derivatives for Dynamic Nuclear Polarization in Liquids: The Role of Rotational Diffusion
}

\author{
M. Levien, M. Hiller, I. Tkach, M. Bennati, and T. Orlando* \\ Cite This: J. Phys. Chem. Lett. 2020, 11, 1629-1635 \\ Read Online
}

ABSTRACT: Polarization transfer efficiency in liquid-state dynamic nuclear polarization (DNP) depends on the interaction between polarizing agents (PAs) and target nuclei modulated by molecular motions. We show how translational and rotational diffusion differently affect the DNP efficiency. These contributions were disentangled by measuring ${ }^{1} \mathrm{H}$ DNP enhancements of toluene and chloroform doped with nitroxide derivatives at $0.34 \mathrm{~T}$ as a function of either the temperature or the size of the PA. The results were employed to analyze ${ }^{13} \mathrm{C}$-DNP data at higher fields, where the polarization transfer is also driven by the Fermi contact interaction. In this case, bulky nitroxide PAs perform better than the small TEMPONE radical due to structural fluctuations of the ring conformation. These findings will help in designing PAs with features specifically optimized for liquid-state DNP at various magnetic fields.

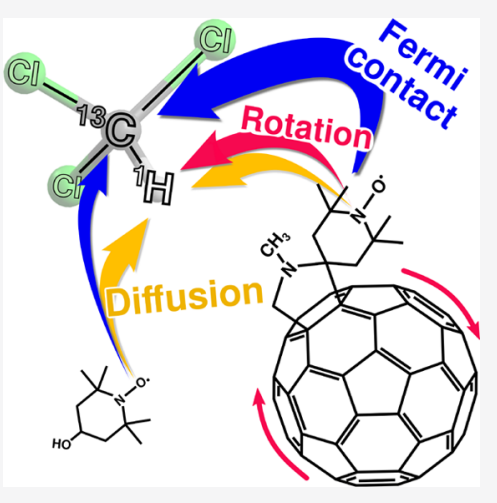

$\mathrm{D}$ ynamic nuclear polarization (DNP) is a class of methods developed to overcome the low sensitivity issue affecting nuclear magnetic resonance (NMR) measurements. They rely on the transfer of the high spin polarization of a polarizing agent (PA) to coupled target nuclei via microwave (MW) irradiation. ${ }^{1}$ DNP in liquids at room temperature, often known as Overhauser DNP, ${ }^{2,3}$ is a spin relaxation mechanism driven by the time modulation of the hyperfine coupling between an unpaired electron and a target nuclear spin through molecular motions. This technique allows for the direct polarization of target nuclei and therefore has the potential to become a precious tool for routine NMR spectroscopy. 4

In the past decade, DNP in liquids on ${ }^{1} \mathrm{H}$ nuclei has been extensively studied at various magnetic fields. ${ }^{6-9}$ In this case, dipolar relaxation modulated by molecular diffusion provides the main mechanism for the polarization transfer. ${ }^{10-13}$ However, the efficiency of such a process decreases with rising magnetic field strength and limits the enhancements to $\epsilon \lesssim 10^{2}$ at $9.4 \mathrm{~T}$ on DNP-optimized instruments. ${ }^{14}$

More recently, larger enhancements have been observed for ${ }^{13} \mathrm{C}$ nuclei, ${ }^{15-17}$ reaching $\epsilon>400$ at $9.4 \mathrm{~T}$ and $\epsilon>20$ at $14.1 \mathrm{~T}$. For ${ }^{13} \mathrm{C}$, the polarization transfer is driven by a mixture of dipolar relaxation and the counteracting scalar relaxation, based on the Fermi contact interaction. The latter is modulated by fast molecular collisions ${ }^{15,17}$ (from subpicoseconds to picoseconds) and has been predicted to be the most efficient contribution. ${ }^{15}$

Small nitroxide radicals have often been chosen as PAs for liquid DNP thanks to their efficiency, which is usually attributed to fast diffusion rates and to the localized electron spin density. Other PAs have not been systematically tested, and only a few studies report on them, ${ }^{18-22}$ whereas PA optimization is a thriving field in solid-state DNP. ${ }^{23,24}$ Recently, we reported 50\% larger ${ }^{13} \mathrm{C}$ scalar enhancements when fullerene nitroxides (FNs) are used as PAs in comparison with the TEMPO radical. ${ }^{17}$ This unexpected observation still lacks a satisfactory explanation and shows how the current understanding of the scalar relaxation is limited. Therefore, it is still difficult to design a PA whose features are specifically tuned for boosting the DNP efficiency in liquids.

We aim at elucidating the mechanisms contributing to the polarization transfer when the mobility of the PA changes. DNP enhancements on ${ }^{1} \mathrm{H}$ nuclei were measured at low field as a function of the temperature and the size of the PA. In this way, we separately targeted translational diffusion and rotational diffusion, disentangling their impact on the polarization transfer mechanism. DNP enhancements were then measured on ${ }^{13} \mathrm{C}$ nuclei at higher fields. In this case, the polarization transfer is driven by a complex interplay of translational diffusion, rotational diffusion, and the Fermi contact. Semiclassical theory and atomistic simulations were used to investigate how the peculiar differences in the structural dynamics of FN and small nitroxide radicals affect the DNP efficiency.

To analyze the DNP mechanism, it is essential to access the DNP coupling factor, $\xi$, which describes the efficiency of the relaxation processes driving the polarization transfer. ${ }^{25} \xi$ can be

Received: November 14, 2019

Accepted: January 31, 2020

Published: January 31, 2020 

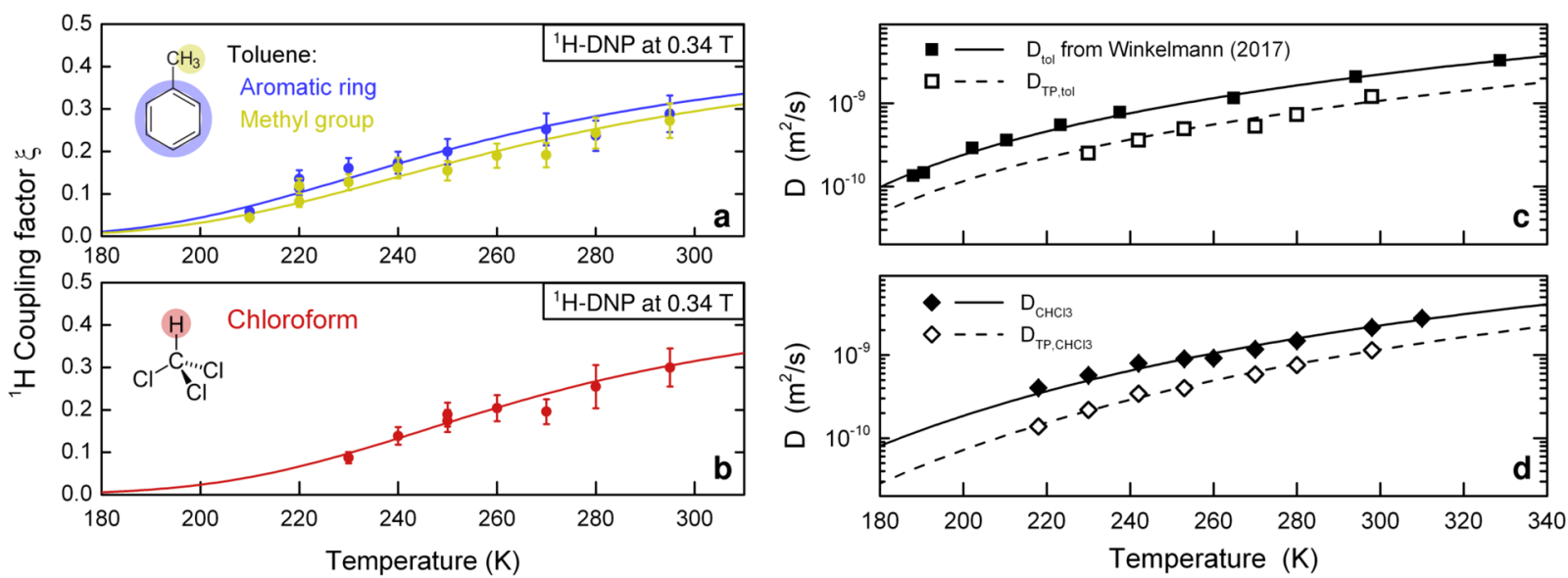

Figure 1. $(\mathrm{a}, \mathrm{b}){ }^{1} \mathrm{H}$ coupling factor $\xi$ for toluene and chloroform doped with TP as a function of temperature. The values at room temperature agree with the predictions from molecular dynamics calculations. ${ }^{11,30}$ Fits (solid lines) were performed with eq 2 , where $k_{\text {rot }} \approx 0$. (c,d) Self-diffusion coefficient of solvent $\left(D_{s}\right)$ and TP in the solvent $\left(D_{\mathrm{TP}, \mathrm{s}}\right)$ as a function of temperature for toluene and chloroform. Lines are fits performed with a Speedy-Angell power law (toluene) and the Arrhenius function (chloroform) (Supporting Information).

calculated from the NMR enhancement $\epsilon$ with the Overhauser equation $^{3}$

$$
\epsilon=1-f s_{\text {eff }} \xi \frac{\left|\gamma_{\mathrm{e}}\right|}{\gamma_{\mathrm{n}}}
$$

where $\gamma_{\mathrm{e}}$ and $\gamma_{\mathrm{n}}$ are the electron and the nuclear gyromagnetic ratio, respectively. The leakage factor $(f)$ and the effective saturation factor $\left(s_{\text {eff }}\right)$ were determined independently via nuclear $T_{1}$ relaxation measurements and ELDOR experiments, ${ }^{26}$ respectively (Supporting Information).

${ }^{1} \mathrm{H}$-DNP experiments were performed at $0.34 \mathrm{~T}$ in the temperature range of $200-295 \mathrm{~K}$ for toluene and $220-295 \mathrm{~K}$ for chloroform, both doped with $\sim 1 \mathrm{mM}$ of TEMPOL radical (TP). The coupling factor $\xi_{\mathrm{H}}$ calculated from eq 1 decreases monotonically for decreasing temperatures for both toluene and chloroform, and it is positive over the whole temperature range (Figure 1a,b). Because $\xi_{\mathrm{H}}>0$, the polarization transfer is dominated by dipolar relaxation, whereas the scalar contribution for ${ }^{1} \mathrm{H}$-DNP is negligible. ${ }^{10,22}$ Employing semiclassical relaxation theory, ${ }^{10}$ the coupling factor for dipolar relaxation can be calculated considering both translational diffusion and molecular rotations ${ }^{10}$

$$
\xi=\frac{5}{7}\left(1-\frac{3 k_{\mathrm{D}} J_{\mathrm{D}}\left(\omega_{\mathrm{n}}, \tau_{\mathrm{D}}\right)+3 k_{\mathrm{rot}} J_{\mathrm{rot}}\left(\omega_{\mathrm{n}}, \tau_{\mathrm{c}}\right)}{R_{1, \mathrm{D}}+R_{1, \mathrm{rot}}}\right)
$$

where $\omega_{\mathrm{n}}$ is the proton Larmor frequency. $R_{1, i}$ is the nuclear relaxation rate, $k_{i}$ is the amplitude, and $J_{i}$ is the spectral density of the translational diffusion ("D") and the rotational contribution ("rot"), respectively (Supporting Information). The spectral density $J_{\mathrm{D}}\left(\omega_{n}, \tau_{\mathrm{D}}\right)$, where $\tau_{\mathrm{D}}$ is the correlation time, is described by the force-free hard-sphere model (ffHS) for translational diffusion. $^{27,28}$ The rotational component can be included phenomenologically with a Lorentzian spectral density $J_{\text {rot }}\left(\omega_{n}, \tau_{c}\right)$, with $\tau_{\mathrm{c}}$ as the rotational correlation time. ${ }^{13,29}$

As previously predicted for water ${ }^{10,31}$ and observed in organic solvents, ${ }^{32}$ the coupling factor $\xi_{1}$ at room temperature for small organic radicals is mainly dependent on translational diffusion, and including a rotational contribution introduces an overparameterization. ${ }^{33,34}$ Therefore, as a first approximation, we assume $k_{\text {rot }}=0$ for $\mathrm{TP}$, leaving only the translational diffusion component in eq 2 . The correlation time is defined as $\tau_{\mathrm{D}}=r_{\mathrm{D}}^{2} /$
$\left(D_{\mathrm{s}}+D_{\mathrm{r}, \mathrm{s}}\right)$, where $r_{\mathrm{D}}$ is the distance of the closest approach and $D_{\mathrm{s}}$ and $D_{\mathrm{r}, \mathrm{s}}$ are the self-diffusion coefficients of the solvent and of the radical in the solvent, respectively. Therefore, it is necessary to determine $D_{\mathrm{s}}$ and $D_{\mathrm{r}, \mathrm{s}}$ over a wide range of temperatures.

Figure $1 \mathrm{c}, \mathrm{d}$ shows $D_{\mathrm{s}}$ and $D_{\mathrm{r}, \mathrm{s}}$ of the investigated solvents as a function of temperature. $D_{\mathrm{TP} \text {,tol }}, D_{\mathrm{CHCl}_{3}}$, and $\mathrm{D}_{\mathrm{TP}, \mathrm{CHCl}_{3}}$ were measured with pulsed-field gradient NMR (Supporting Information), whereas $D_{\text {tol }}$ is reproduced from the literature. ${ }^{35}$

With $D_{\mathrm{s}}$ and $D_{\mathrm{r}, \mathrm{s}}$, the fit of the coupling factor $\xi_{1}$ to eq 2 requires only one free parameter, which is the distance of closest approach, $r_{\mathrm{D}}$, that is, the average distance over the different directions of approach between the PA and the target molecule. ${ }^{10}$ The fits are in good agreement with the experimental data (Figure 1) and confirm that a slower translational diffusion reduces the coupling factor $\xi_{1}{ }_{\mathrm{H}}$. The best-fit parameters are $r_{\mathrm{D}}^{\text {ring }}=3.5 \AA$ and $r_{\mathrm{D}}^{\text {methyl }}=3.9 \AA$ for toluene and $r_{\mathrm{D}}=3.65 \AA$ for chloroform, respectively. The trend $\xi_{1} \mathrm{H} g>\xi^{1} \mathrm{H}$ hyl that is experimentally observed for toluene over the whole temperature range implies $r_{\mathrm{D}}^{\text {ring }}<r_{\mathrm{D}}^{\text {methyl }}$. Interestingly, the geometries of the $\mathrm{TP}$-toluene complexes optimized via density functional theory (DFT) calculations reflect the same relation (Supporting Information).

Besides the translational diffusion, we aimed to selectively target the influence of molecular rotation by testing a series of bulkier nitroxide derivatives as PAs in both chloroform and toluene. They consist of $3 \beta$-doxyl-5 $\alpha$-cholestane (TP-CLST) and FNs, that is, nitroxide radicals functionalized with fullerene $\mathrm{C}_{60}$, having additional side chains (up to three) that improve the solubility and increase the rotational correlation time ${ }^{22}$ (Figure $2 a)$. All selected nitroxide derivatives have the spin density localized on the NO group. Therefore, the hyperfine coupling that drives the polarization transfer is not different from the TP radical. However, their increasing molecular size affects the rotational correlation time of the PA in the solvent, which ranges from $\tau_{\mathrm{c}}^{\mathrm{EPR}} \approx 6$ ps for the TP radical to $\tau_{\mathrm{c}}^{\mathrm{EPR}} \approx 450$ ps for larger FNs, as obtained from continuous-wave electron paramagnetic resonance (CW-EPR) measurements at $9 \mathrm{GHz}$ (Table 1 and Supporting Information).

The coupling factor $\xi_{1}$ of chloroform doped with nitroxide derivatives $(c \approx 1 \mathrm{mM})$ was measured at $0.34 \mathrm{~T}$ at room temperature, whereas the values for toluene at the same field were previously reported. $^{22}$ As shown in Figure 2, $\xi_{1}{ }_{\mathrm{H}}$ decreases 


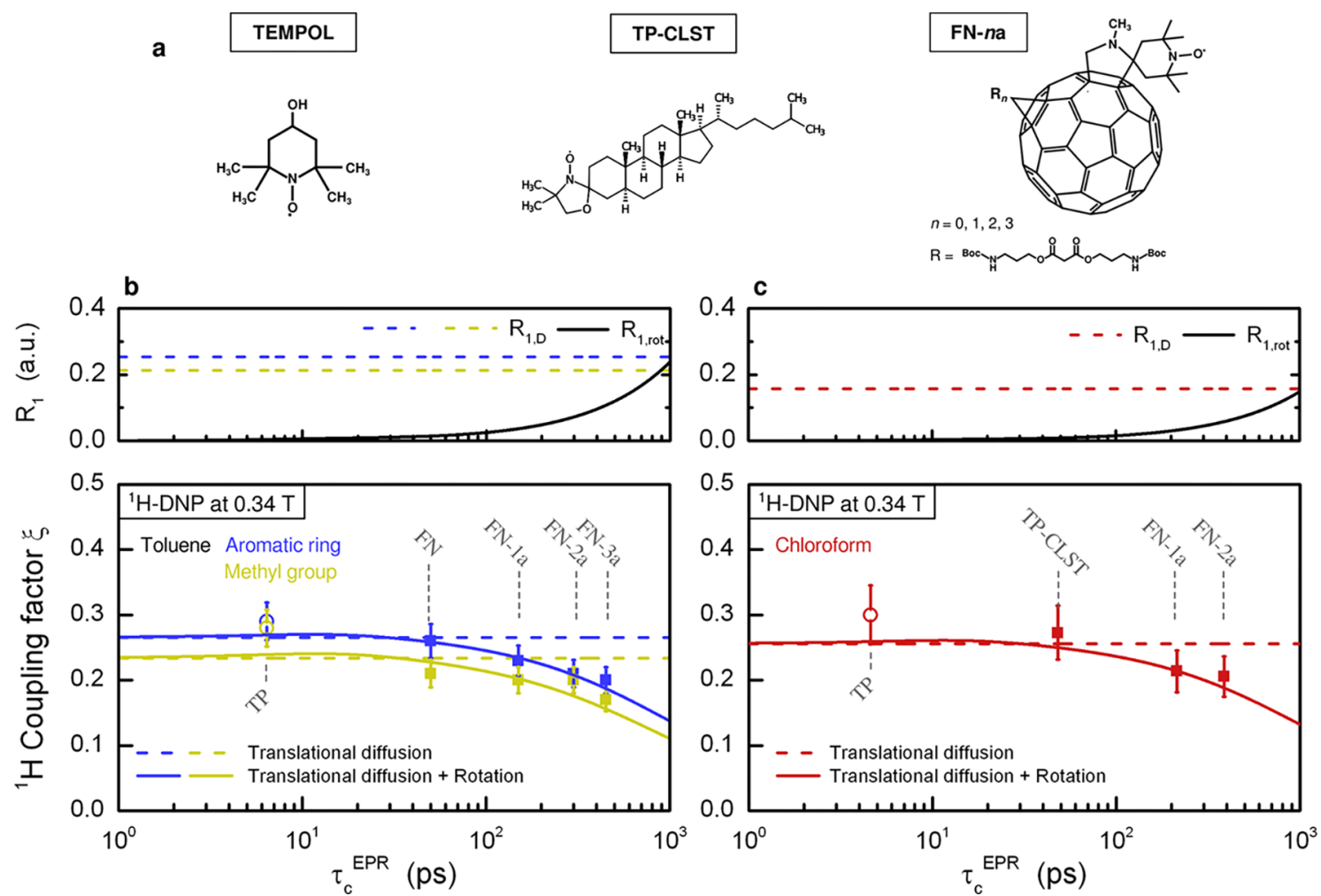

Figure 2. (a) Structure of nitroxide radical and nitroxide derivatives used as PAs. (b,c, bottom) Coupling factor $\xi^{1}{ }_{\mathrm{H}}$ of toluene $\mathrm{e}^{22}$ and chloroform doped with nitroxide derivatives plotted as a function of the rotational correlation time $\tau_{\mathrm{c}}^{\mathrm{EPR}}$ of the PA. Radical concentration was $c \approx 1.5 \mathrm{mM}$ for toluene and $c \approx 1 \mathrm{mM}$ for chloroform. $\xi$ was simulated with eq 2 without (dashed lines) and with the rotational contribution (solid lines). (b,c, top) Relaxation rates for translational diffusion $\left(R_{1, \mathrm{D}}\right)$ and rotation $\left(R_{1, \text { rot }}\right)$ as a function of $\tau_{\mathrm{c}}$.

Table 1. Correlation Times (in ps) for Translational Diffusion $\left(\tau_{\mathrm{D}}\right)$ and Rotation $\left(\tau_{\mathfrak{c}}^{\mathrm{EPR}}\right)$ in Toluene and Chloroform Doped with TP and Nitroxide Derivatives

\begin{tabular}{|c|c|c|c|c|c|c|c|c|}
\hline \multicolumn{5}{|c|}{ toluene with TEMPOL } & \multicolumn{4}{|c|}{ chloroform with TEMPOL } \\
\hline$T(\mathrm{~K})$ & $\tau_{\mathrm{D}}^{\text {ring }}$ & $\tau_{\mathrm{D}}^{\text {methyl }}$ & $\tau_{\mathrm{c}}^{\mathrm{EPR}}$ & $\tau_{\mathrm{D}} / \tau_{\mathrm{c}}^{\mathrm{EPR}}$ & $T(\mathrm{~K})$ & $\tau_{\mathrm{D}}$ & $\tau_{\mathrm{c}}^{\mathrm{EPR}}$ & $\tau_{\mathrm{D}} / \tau_{\mathrm{c}}^{\mathrm{EPR}}$ \\
\hline 200 & 344 & 427 & 16.2 & $>21$ & 220 & 256 & 30 & 8.5 \\
\hline 240 & 108 & 134 & 10.4 & $>10$ & 240 & 142 & 14 & 10 \\
\hline 270 & 59 & 74 & 7.4 & $>7.9$ & 270 & 69 & 7.3 & 9.5 \\
\hline 297 & 38 & 47 & 6.4 & $>5.9$ & 297 & 40 & 4.6 & 8.7 \\
\hline \multicolumn{5}{|c|}{ toluene with nitroxide derivatives } & \multicolumn{4}{|c|}{ chloroform with nitroxide derivatives } \\
\hline sample & $\tau_{\mathrm{D}}^{\text {ring }}$ & $\tau_{\mathrm{D}}^{\text {methyl }}$ & $\tau_{\mathrm{c}}^{\mathrm{EPR}(\mathrm{a})}$ & $\tau_{\mathrm{D}} / \tau_{\mathrm{c}}^{\mathrm{EPR}}$ & sample & $\tau_{\mathrm{D}}$ & $\tau_{\mathrm{c}}^{\mathrm{EPR}}$ & $\tau_{\mathrm{D}} / \tau_{\mathrm{c}}^{\mathrm{EPR}}$ \\
\hline FN & 57 & 71 & 50 & $<1.4$ & TP-CLST & 61 & 19 & 3.2 \\
\hline FN-1a & 57 & 71 & 150 & $<0.5$ & FN-1a & 61 & 214 & 0.3 \\
\hline FN-2a & 57 & 71 & 300 & $<0.24$ & FN-2a & 61 & 385 & 0.16 \\
\hline FN-3a & 57 & 71 & 450 & $<0.16$ & & & & \\
\hline
\end{tabular}

${ }^{a}$ Error on $\tau_{\mathrm{c}}^{\mathrm{EPR}}$ is $\sim 10 \%$. Error on $\tau_{\mathrm{D}}$ is $<15 \%$. Data from Enkin et al., 2015. ${ }^{22}$

for larger $\tau_{\mathrm{c}}^{\mathrm{EPR}}$ and so does the DNP efficiency. The rationale for describing the polarization transfer requires an interplay of translational diffusion, governed by $\tau_{\mathrm{D}}$, and rotational motion, characterized by the rotational correlation time, $\tau_{\mathrm{c}}$. The translational diffusion of solvent molecules nearby the NO group of the PA remains almost unchanged compared with free TP because the accessibility to the radical is not hampered. ${ }^{21}$ Therefore, the distance of closest approach, $r_{\mathrm{D}}$, in the ffHS model is considered to be independent of the particular derivative under study. However, because of the larger molecular size, the diffusion coefficient of nitroxide derivatives in the solvent $\left(D_{\mathrm{r}, \mathrm{s}}\right)$ is expected to be much smaller than $D_{\mathrm{TP}, \mathrm{s}}$. A reasonable approximation consists of choosing $D_{\mathrm{r}, \mathrm{s}} \approx 0$ for nitroxide derivatives, which leads to $\tau_{\mathrm{D}} \approx r_{\mathrm{D}}^{2} / D_{\mathrm{s}}$ (Table 1). The rotational contribution of the dipolar relaxation becomes larger for slower rotations, as previously observed in high-viscosity liquids. $^{29,36}$ This is the case in FN-na (where $n$ is the number of adducts), with rotational correlation times $\tau_{\mathrm{c}}^{\mathrm{EPR}}$ larger than $\tau_{\mathrm{D}}$ (Table 1). The rotational dynamics of the solvent molecules are much faster, being $\tau_{\mathrm{c}}<2$ ps for both toluene ${ }^{37}$ and chloroform, ${ }^{38}$ meaning that their contribution can be neglected at this magnetic field $\left(\tau_{\mathrm{c}} \omega_{\mathrm{n}} \ll 1\right)$. Therefore, the spectral density $J_{\text {rot }}\left(\omega_{i}, \tau_{\mathrm{c}}\right)=2 \tau_{\mathrm{c}} /\left(1+\tau_{\mathrm{c}}^{2} \omega_{i}^{2}\right)$ can be calculated assuming $\tau_{\mathrm{c}} \approx$ $\tau_{\mathrm{c}}^{\mathrm{EPR}}$.

The data in Figure 2 were fitted to eq 2, where the sole free parameter is the amplitude of the rotational contribution $k_{\text {rot }}$ whereas $k_{\mathrm{D}}$ is determined by $\tau_{\mathrm{D}}$ and the radical concentration $c$ (Supporting Information). The model fits the experimental data 
(Figure 2) when $k_{\text {rot }}=0.8 \times 10^{8}$ for toluene and $k_{\text {rot }}=0.5 \times 10^{8}$ for chloroform. $k_{\text {rot }}$ is similar for the two investigated solvents, and thus a bound state due to secondary interactions, such as $\pi$ stacking or halogen-bond-like interactions between the PA and the target molecule, is unlikely. Therefore, the rotational contribution is determined mainly by the choice of the PA. In particular, we note that in the limit of either fast rotating PAs $\left(\tau_{\mathrm{c}}\right.$ $\left.<20 \times 10^{-12} \mathrm{~s}\right)$ or immobilized PAs $\left(\tau_{\mathrm{c}}>10^{-5} \mathrm{~s}\right)$, this contribution is ineffective (Supplementary Figure S10).

The effect of bulkier nitroxide derivatives used as PAs in liquid-state DNP was also investigated for ${ }^{13} \mathrm{C}$ as target nuclei. In this case, the scalar relaxation effectively contributes to the polarization transfer. Nitroxide derivatives TP-CLST, FN-1a, and $\mathrm{FN}-2 \mathrm{a}$ were tested as PAs in ${ }^{13} \mathrm{CCl}_{4}$ and ${ }^{13} \mathrm{CHCl}_{3}(c \approx 10-$ $20 \mathrm{mM}$ ), whereas $\mathrm{FN}$ and $\mathrm{FN}-3 \mathrm{a}$ showed instability in those solvents. DNP on ${ }^{13} \mathrm{C}$ nuclei was performed at $1.2 \mathrm{~T}$ rather than at $0.34 \mathrm{~T}$ due to better NMR sensitivity. The experimental $\xi^{13} \mathrm{C}$ values are reported in Figure 3 and show an increase in absolute

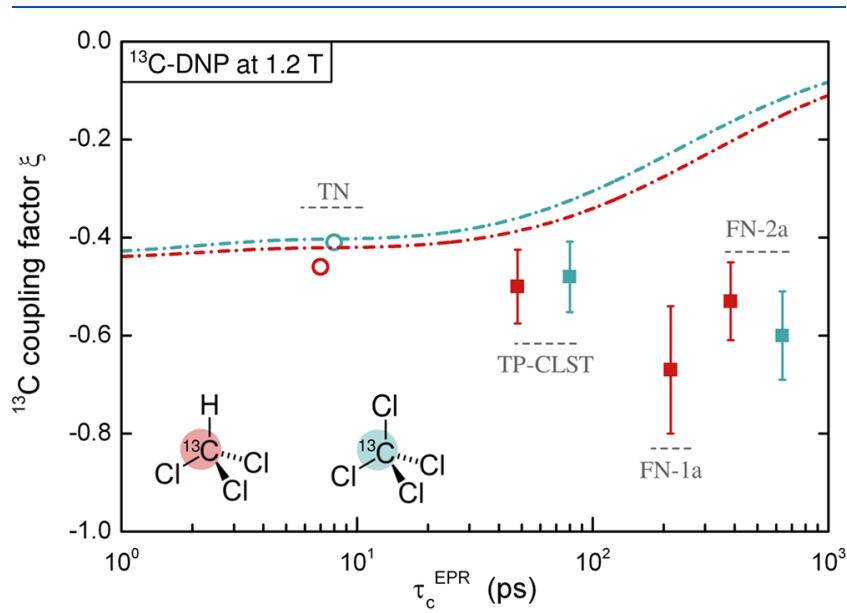

Figure 3. $\xi^{13} \mathrm{C}$ from ${ }^{13} \mathrm{C}$-DNP at $1.2 \mathrm{~T}$ on ${ }^{13} \mathrm{CCl}_{4}$ and ${ }^{13} \mathrm{CHCl}_{3}$ doped with nitroxide derivatives. The prediction (dashed-dotted line) has been calculated with eq 4 considering the contributions to the relaxation rates from the translational diffusion $\left(R_{1, \mathrm{D}}\right)$, the contact scalar interaction $\left(R_{1, \text { cont } 1}\right)$, and the rotation $\left(R_{1, \text { rot }}\right)$.

value with the molecular size. This translates into higher DNP efficiency for larger PAs: Specifically, up to $50 \%$ larger ${ }^{13} \mathrm{C}$ enhancements were observed for FN-2a in comparison with the nitroxide radical TEMPONE (TN). ${ }^{17}$ However, the dependency of the dipolar contribution on $\tau_{\mathrm{c}}^{\mathrm{EPR}}$ does not account for such an effect, as discussed in the following paragraph.

The coupling factors $\xi^{13} \mathrm{C}$ for TN in $\mathrm{CCl}_{4}$ and $\mathrm{CHCl}_{3}$ were modeled using a combination of relaxation rates driven by the translational diffusion $\left(R_{1, \mathrm{D}}\right.$ from the ffHS model) and the scalar relaxation arising from the Fermi contact $\left(R_{1, \text { cont }}\right)$, as reported in previous studies. ${ }^{15,17} R_{1, \text { cont }}$ is described by the Pulse model for random molecular collisions, ${ }^{39}$ which comprises different types $i$ of contact, each one characterized by a duration, $2 \tau_{i}$, and a collision frequency, $1 / \tau_{\mathrm{p}, i}$

$$
R_{1, \text { cont }}=\sum_{i} R_{1, \operatorname{cont}(i)}=\frac{2}{3} S(S+1) \sum_{i} F_{i}\left(\tau_{i} \cdot \exp \left(-\tau_{i} \omega_{\mathrm{e}}\right)\right)^{2}
$$

where $F_{i}=\left\langle A_{i}\right\rangle^{2} /\left(\hbar^{2} \tau_{\mathrm{p}, i}\right)$, and $\left\langle A_{i}\right\rangle^{2}$ is the mean- square amplitude over time of the scalar interaction. For $\mathrm{CCl}_{4}$ and $\mathrm{CHCl}_{3}$, the scalar coupling is modulated mainly by fast molecular collisions with a duration of $\tau_{1} \approx 0.5$ ps. ${ }^{15,17}$ The scalar contribution is included in eq 2 as follows ${ }^{10}$

$$
\begin{aligned}
\xi= & \frac{5}{7}\left(1-\frac{3 k_{\mathrm{D}} J_{\mathrm{D}}\left(\omega_{\mathrm{n}}, \tau_{\mathrm{D}}\right)+3 k_{\text {rot }} J_{\mathrm{rot}}\left(\omega_{\mathrm{n}}, \tau_{\mathrm{c}}\right)}{R_{1}}\right) \\
& -\frac{12}{7}\left(\frac{R_{1, \text { cont }}}{R_{1}}\right)
\end{aligned}
$$

where $R_{1}=R_{1, \mathrm{D}}+R_{1, \text { rot }}+R_{1, \text { cont }}$. Equation 4 can be used to predict $\xi^{13} \mathrm{C}$ for nitroxide derivatives at $1.2 \mathrm{~T}$. The component $R_{1, \mathrm{D}}$ was previously parametrized for the $\mathrm{TN}$ radical ${ }^{17}$ and has now been rescaled for the diffusion of larger PAs (Supplementary Table S.IX). $R_{1, \text { cont }}$ was modeled as in ref 17 . The amplitude of the rotational component was fixed to $k_{\text {rot }}=5 \times 10^{8}$ for both solvents, as determined from the low-field analysis of chloroform, and rescaled for $c \approx 10 \mathrm{mM}$. However, the prediction of $\xi^{13} \mathrm{C}$ calculated with eq 4 does not fit the experimental data, as shown in Figure 3. This implies that for nitroxide derivatives, the Fermi contact hyperfine coupling between the NO group and the target molecule could be subject to additional modulations with time scales different from the one of random molecular collisions.

To quantitatively access the time scales at which the hyperfine coupling is modulated, we also analyzed $\xi^{13} \mathrm{C}$ values reported in a previous study ${ }^{17}$ for different magnetic fields (1.2, 9.4, and 14.1 T). FN-2a in $\mathrm{CCl}_{4}$ was chosen as a study system due to its good DNP performance at low fields. Whereas the efficiency of FN-2a as a PA is higher than that of TN in a low field $\left(\left|\xi_{{ }^{13} \mathrm{CN}}^{\mathrm{FN}}\right|>\right.$ $\left.\left|\xi_{13}^{\mathrm{TN}}\right|\right)$, the situation is reversed at high fields $\left(\left|\xi_{13}^{\mathrm{FN} 2} \mathrm{C}\right| \ll\left|\xi_{13}^{\mathrm{TN}} \mathrm{C}\right|\right)$, where the enhancements in ${ }^{13} \mathrm{CCl}_{4}$ at $9.4 \mathrm{~T}$ are $\epsilon \approx 10$ and 430 for FN-2a and TN, ${ }^{17}$ respectively. $\xi_{13}^{\mathrm{FN} 2 \mathrm{C}}$ was simulated with eq 4 with the components $R_{1, \mathrm{D}}, R_{1, \text { rot }}$ and $R_{1, \text { cont }}$ (Table 2), where now the scalar part considers not only one but rather two types of collisions $\left(R_{1, \text { cont }}=R_{1, \text { cont } 1}+R_{1, \text { cont } 2}\right)$. Despite the few

\begin{tabular}{|c|c|c|c|c|c|c|c|}
\hline \multirow[b]{2}{*}{ solvent } & \multirow[b]{2}{*}{ radical } & \multicolumn{2}{|c|}{ rotation } & \multicolumn{2}{|c|}{ contact 1} & \multicolumn{2}{|c|}{ contact 2} \\
\hline & & $k_{\text {rot }}$ & $\tau_{\mathrm{c}}(\mathrm{ps})$ & $\sqrt{F_{1}}$ & $\tau_{1}(\mathrm{ps})$ & $\sqrt{F_{2}}$ & $\bar{\tau}_{2}(\mathrm{ps})^{c}$ \\
\hline $\mathrm{CCl}_{4}$ & $\mathrm{TN}$ & & $7.7^{b}$ & $1.25 \times 10^{12}$ & 0.5 & & \\
\hline $\mathrm{CCl}_{4}$ & $\mathrm{FN}-2 \mathrm{a}$ & $5 \times 10^{8}$ & 637 & $1.25 \times 10^{12}$ & 0.5 & $1.2 \times 10^{12}$ & 3.0 \\
\hline $\mathrm{CHCl}_{3}{ }^{a}$ & $\mathrm{TN}$ & & 4.8 & $1.25 \times 10^{12}$ & 0.5 & & \\
\hline $\mathrm{CHCl}_{3}{ }^{a}$ & FN-2a & $5 \times 10^{8}$ & 385 & $1.25 \times 10^{12}$ & 0.5 & $0.8 \times 10^{12}$ & 3.0 \\
\hline
\end{tabular}

Table 2. Correlation Times and Amplitudes of the Relaxation Contributions Used for Simulating $\xi^{\mathrm{TN}} \mathrm{C}$ (from Ref 17 ) and $\xi^{\mathrm{FN}} \mathrm{C}$ as a Function of the Magnetic Field

${ }^{a}$ For $\mathrm{CHCl}_{3}$, an additional Fermi contact component $R_{1, \mathrm{cont} 1, \mathrm{H}}$ arises from hydrogen-bond-like collisions mediated by the $\mathrm{H}$ atom: The parameters are $\sqrt{F_{1, \mathrm{H}}}=0.5 \times 10^{12} \mathrm{rad} / \mathrm{s}, \tau_{1, \mathrm{H}}=12 \mathrm{ps}$, as reported in ref $17 .{ }^{b}$ Data from Liu et al., $2017 .{ }^{15}{ }^{c} \bar{\tau}_{2}$ is the best-fit value within the range $\tau_{2}=2.0$ to 6.0 ps (Supplementary Figure S11). 

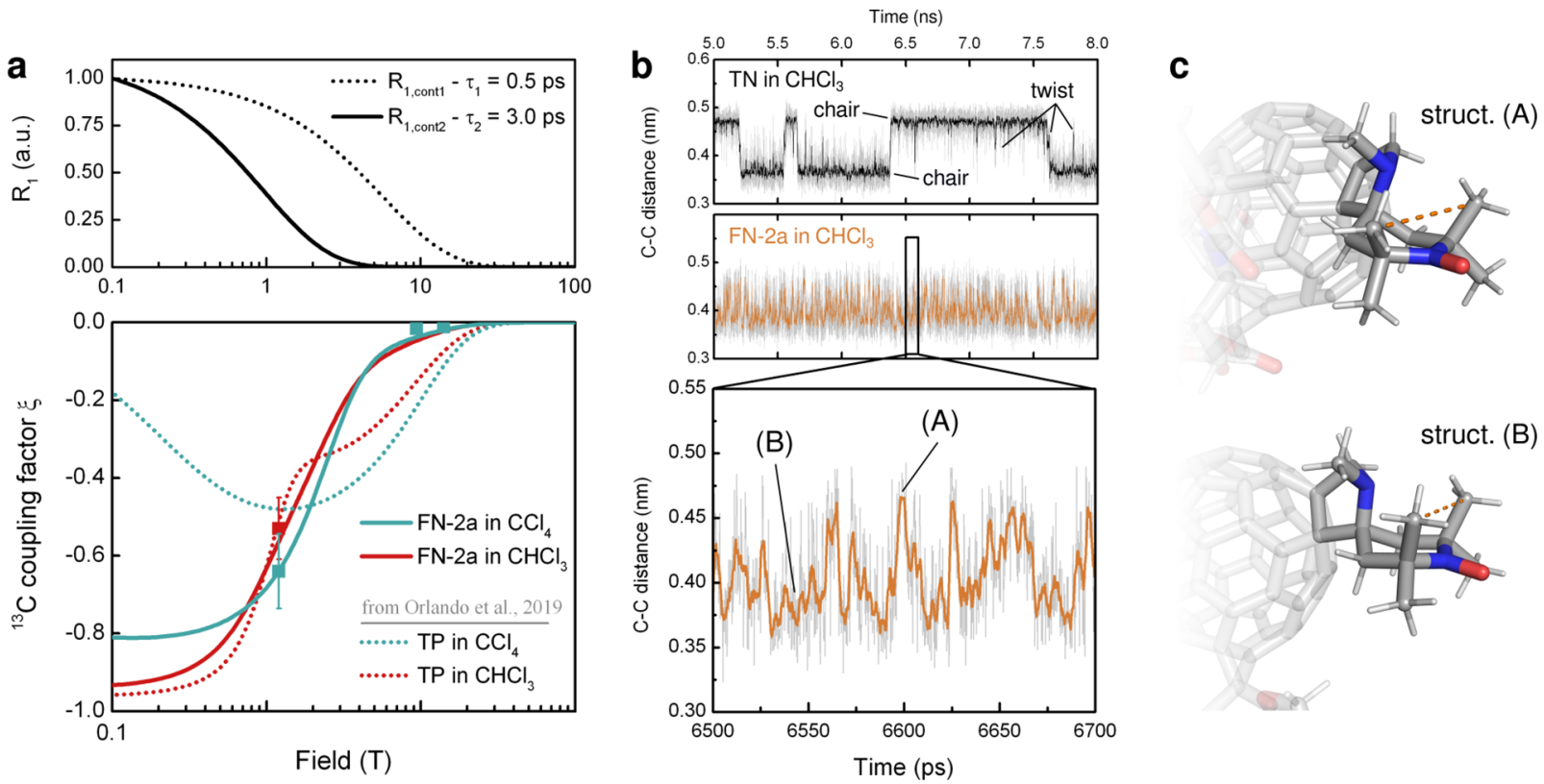

Figure 4. (a) $\xi_{13 \mathrm{C}}^{\mathrm{FN} 2 \mathrm{a}}$ in $\mathrm{CCl}_{4}$ and $\mathrm{CHCl}_{3}$ as a function of the magnetic field: experimental data (squares) and fits with eq 4 (solid lines). The relaxation contributions calculated with the values in Table 2 and normalized are shown in the top panel. Calculations for $\xi_{13}^{\mathrm{TN}} \mathrm{C}$ (dotted lines) are shown for comparison. ${ }^{17}$ (b) Time trace of the $\mathrm{C}-\mathrm{C}$ distance of the methyl groups on one side of the nitroxide ring obtained from MD runs for TN and FN-2a in chloroform (total time $=10 \mathrm{~ns}, T=300 \mathrm{~K}$, integration step $=2 \mathrm{fs}$ ). Dark lines are smoothed data (11 pt moving average). (c) Structural variation observed with the measured distance indicated in orange in FN-2a as extracted from the MD simulation.

experimental data points, the correlation time $\tau_{2}$ of the additional contribution $R_{1, \text { cont2 }}$ can be reasonably estimated due to its large impact on the shape of $\xi$ as a function of the magnetic field (Supplementary Figure S11 and ref 17), whereas the amplitude $F_{2}$ acts as a scaling factor. From this analysis, we obtained $\tau_{2}=2.0-6.0 \mathrm{ps}$, where $\bar{\tau}_{2}=3.0 \mathrm{ps}$ is the best-fit parameter, with an amplitude $F_{2}$ accounting for $\sim 40 \%$ of the total scalar contribution (Table 2 and Figure 4a).

To shine light onto the origin of this collision on a longer time scale, the behavior of nitroxide derivatives was explored by a computational approach combining DFT calculations and molecular dynamics (MD) simulations. Specifically, we focused on possible structural rearrangements of the nitroxide groups capable of modulating the hyperfine interaction. The conformational space of TN and FN-2a was explored by DFT calculations to identify structures corresponding to true energetic minima (Supporting Information). As already known for TN, a chairlike conformation constitutes the energetic minimum, whereas a twist structure is higher in energy $(\sim 2.9 \mathrm{~kJ} / \mathrm{mol})$ yet still accessible at $T=300 \mathrm{~K}$. For FN-2a, the asymmetry of the linker increases the number of possible conformations, but only one chairlike conformation was identified as the most favorable. In contrast with TN, other arrangements, such as the "boat" and, higher in energy, the twist, are inaccessible for FN-2a at room temperature.

The dynamics of both PAs were probed by MD simulations in $\mathrm{CHCl}_{3}$ using GROMACS $2018.4^{40}$ and a set of previously reported parameters for the nitroxide radicals. ${ }^{41}$ The results are summarized in Figure $4 b$, where the $\mathrm{C}-\mathrm{C}$ distance of the methyl groups on one side of the nitroxide ring is used as a descriptor of the corresponding ring conformation. For TN, the expected interchange of chair conformations via twist intermediates is observed (Figure 4b). In contrast, FN-2a shows a stable chair conformation that remains unchanged at the simulation temperature in the investigated time frame, in agreement with
DFT predictions. However, a different type of structural variation is observable for $\mathrm{FN}-2 \mathrm{a}$, best described as the transformation of a chair to a half-chair conformation of the six-membered ring (Figure 4c). With respect to the DFT results, this corresponds to a transition state between the two conformers of lowest energy, that is, a chair and a "boat" (Supporting Information). Notably, this structural fluctuation happens on a time scale of few picoseconds (Figure $4 \mathrm{~b}$ ), which correlates well with the one extracted from the $\xi^{13} \mathrm{C}$ experimental data (collision duration $2 \cdot \tau_{2}=4.0-12.0 \mathrm{ps}$ ). This suggests that the rearrangement of the methyl groups could effectively contribute to the modulation of the Fermi contact between the NO group and the target molecule, thus affecting the DNP efficiency.

In conclusion, we have explored how the mobility of the PA affects the efficiency of DNP in liquids. Translational diffusion and rotational diffusion can be disentangled by independently manipulating the temperature and the molecular size of the PA, respectively. In the case of ${ }^{1} \mathrm{H}-\mathrm{DNP}$, where the polarization transfer is mainly driven by dipolar relaxation, fast rotating small molecules or immobilized radicals are the best choices as PAs. The situation is more complex for DNP on ${ }^{13} \mathrm{C}$ nuclei when FNs are used as PAs. The Fermi contact interaction between the target molecule and the PA is modulated at least on two different time scales $\left(2 \tau_{1} \approx 1\right.$ ps and $\left.2 \tau_{2} \approx 6 \mathrm{ps}\right)$, which are determined by the molecular collision and, as suggested here, by conformational changes of the PA. Our findings show how rotational diffusion and structural reorientations affect the DNP performances at specific magnetic fields. We foresee that these results will help in designing optimal PAs/target systems for efficient DNP in liquids and eventually boost new applications of NMR spectroscopy. 


\section{ASSOCIATED CONTENT}

\section{(s) Supporting Information}

The Supporting Information is available free of charge at https://pubs.acs.org/doi/10.1021/acs.jpclett.0c00270.

Sample preparation, experimental methods, CW-EPR measurements and fit, PFG-NMR measurements, ${ }^{1} \mathrm{H}-$ $\mathrm{DNP}$ and ${ }^{13} \mathrm{C}$-DNP results, analytical models for the coupling factor, and DFT and MD simulation parameters for $\mathrm{TN}$ and $\mathrm{FN}-2 \mathrm{a}$ (PDF)

\section{AUTHOR INFORMATION}

\section{Corresponding Author}

T. Orlando - Research Group EPR Spectroscopy, Max Planck Institute for Biophysical Chemistry, Göttingen 37077, Germany; ๑ orcid.org/0000-0001-7261-2119; Email: tomas.orlando@ mpibpc.mpg.de

\section{Authors}

M. Levien - Research Group EPR Spectroscopy, Max Planck Institute for Biophysical Chemistry, Göttingen 37077, Germany; Department of Chemistry, Georg-August University, Göttingen 37077, Germany

M. Hiller - Research Group EPR Spectroscopy, Max Planck Institute for Biophysical Chemistry, Göttingen 37077, Germany

I. Tkach - Research Group EPR Spectroscopy, Max Planck Institute for Biophysical Chemistry, Göttingen 37077, Germany

M. Bennati - Research Group EPR Spectroscopy, Max Planck Institute for Biophysical Chemistry, Göttingen 37077, Germany; Department of Chemistry, Georg-August University, Göttingen 37077, Germany; 이이이.org/0000-0001-6784-7845

Complete contact information is available at:

https://pubs.acs.org/10.1021/acs.jpclett.0c00270

\section{Notes}

The authors declare no competing financial interest.

\section{ACKNOWLEDGMENTS}

Financial support has been provided by the Max Planck Society. M.L. acknowledges IMPRS-PBCS of the Max Planck Society for funding. We acknowledge Maik Reinhard for conducting ${ }^{1} \mathrm{H}$ DNP experiments. Daniel Sieme and Niels Karschin are acknowledged for the assistance with PFG-NMR measurements. We thank Aljaz Godec for fruitful discussions and the Synthetic Chemistry facility at MPI-BPC for the synthesis of FN samples.

\section{REFERENCES}

(1) Ardenkjaer-Larsen, J. H.; et al. Facing and Overcoming Sensitivity Challenges in Biomolecular NMR Spectroscopy. Angew. Chem., Int. Ed. 2015, 54, 9162-9185.

(2) Overhauser, A. W. Polarization of nuclei in metals. Phys. Rev. 1953, 92, 411-415.

(3) Hausser, K.; Stehlik, D. In Advances in Magnetic Resonance; Waugh, J. S., Ed.; Academic Press, 1968; Vol. 3, pp 79-139.

(4) Denysenkov, V. P.; Prisner, T. F. Liquid-state Overhauser DNP at high magnetic fields. eMagRes 2019, 8, 41-54.

(5) Bennati, M.; Orlando, T. Overhauser DNP in liquids on ${ }^{13} \mathrm{C}$ nuclei. eMagRes 2019, 8, 11-18.

(6) Bennati, M.; Tkach, I.; Türke, M. T. Dynamic nuclear polarization in liquids. Electron Paramagn. Reson. 2010, 22, 155-182.

(7) Franck, J. M.; Pavlova, A.; Scott, J. A.; Han, S. Quantitative cw Overhauser effect dynamic nuclear polarization for the analysis of local water dynamics. Prog. Nucl. Magn. Reson. Spectrosc. 2013, 74, 33-56.
(8) Griesinger, C.; Bennati, M.; Vieth, H. M.; Luchinat, C.; Parigi, G.; Hofer, P.; Engelke, F.; Glaser, S. J.; Denysenkov, V.; Prisner, T. F. Dynamic nuclear polarization at high magnetic fields in liquids. Prog. Nucl. Magn. Reson. Spectrosc. 2012, 64, 4-28.

(9) Van Bentum, J.; Van Meerten, B.; Sharma, M.; Kentgens, A. Perspectives on DNP-enhanced NMR spectroscopy in solutions. J. Magn. Reson. 2016, 264, 59-67.

(10) Bennati, M.; Luchinat, C.; Parigi, G.; Türke, M. T. Water ${ }^{1} \mathrm{H}$ relaxation dispersion analysis on a nitroxide radical provides information on the maximal signal enhancement in Overhauser dynamic nuclear polarization experiments. Phys. Chem. Chem. Phys. 2010, 12, 5902-5910.

(11) Sezer, D. Computation of DNP coupling factors of a nitroxide radical in toluene: seamless combination of $\mathrm{MD}$ simulations and analytical calculations. Phys. Chem. Chem. Phys. 2013, 15, 526-540.

(12) Prisner, T.; Denysenkov, V.; Sezer, D. Liquid state DNP at high magnetic fields: Instrumentation, experimental results and atomistic modelling by molecular dynamics simulations. J. Magn. Reson. 2016, 264, 68-77.

(13) Parigi, G.; Ravera, E.; Bennati, M.; Luchinat, C. Understanding Overhauser Dynamic Nuclear Polarisation through NMR relaxometry. Mol. Phys. 2019, 117, 888-897.

(14) Neugebauer, P.; Krummenacker, J. G.; Denysenkov, V. P.; Parigi, G.; Luchinat, C.; Prisner, T. F. Liquid state DNP of water at 9.2 T: an experimental access to saturation. Phys. Chem. Chem. Phys. 2013, 15, 6049-6056.

(15) Liu, G.; Levien, M.; Karschin, N.; Parigi, G.; Luchinat, C.; Bennati, M. One-thousand-fold enhancement of high field liquid nuclear magnetic resonance signals at room temperature. Nat. Chem. 2017, 9, 676-680.

(16) Dubroca, T.; Wi, S.; van Tol, J.; Frydman, L.; Hill, S. Large Volume Liquid State Scalar Overhauser Dynamic Nuclear Polarization at High Magnetic Field. Phys. Chem. Chem. Phys. 2019, 21, 21200.

(17) Orlando, T.; Dervışoğlu, R.; Levien, M.; Tkach, I.; Prisner, T. F.; Andreas, L. B.; Denysenkov, V. P.; Bennati, M. Dynamic nuclear polarization of ${ }^{13} \mathrm{C}$ nuclei in the liquid state over a $10 \mathrm{~T}$ field range. Angew. Chem., Int. Ed. 2019, 58, 1402-1406.

(18) Wind, R. A.; Ardenkjær-Larsen, J. H. ${ }^{1} \mathrm{H}$ DNP at $1.4 \mathrm{~T}$ of water doped with a triarylmethyl-based radical. J. Magn. Reson. 1999, 141, 347-354.

(19) Loening, N. M.; Rosay, M.; Weis, V.; Griffin, R. G. Solution-state dynamic nuclear polarization at high magnetic field. J. Am. Chem. Soc. 2002, 124, 8808-8809.

(20) Höfer, P.; Parigi, G.; Luchinat, C.; Carl, P.; Guthausen, G.; Reese, M.; Carlomagno, T.; Griesinger, C.; Bennati, M. Field dependent dynamic nuclear polarization with radicals in aqueous solution. J. Am. Chem. Soc. 2008, 130, 3254-3255.

(21) Dollmann, B. C.; Kleschyov, A. L.; Sen, V.; Golubev, V.; Schreiber, L. M.; Spiess, H. W.; Münnemann, K.; Hinderberger, D. Spin-labeled heparins as polarizing agents for dynamic nuclear polarization. ChemPhysChem 2010, 11, 3656-3663.

(22) Enkin, N.; Liu, G.; Gimenez-Lopez, M. d. C.; Porfyrakis, K.; Tkach, I.; Bennati, M. A high saturation factor in Overhauser DNP with nitroxide derivatives: the role of ${ }^{14} \mathrm{~N}$ nuclear spin relaxation. Phys. Chem. Chem. Phys. 2015, 17, 11144-11149.

(23) Kubicki, D. J.; Emsley, L. Improving sensitivity of solid-state NMR spectroscopy by rational design of polarizing agents for dynamic nuclear polarization. Chimia 2017, 71, 190-194.

(24) Casano, G.; Karoui, H.; Ouari, O. Polarizing agents: evolution and outlook in free radical development for DNP. eMagRes 2018, 195208

(25) Ravera, E.; Luchinat, C.; Parigi, G. Basic facts and perspectives of Overhauser DNP NMR. J. Magn. Reson. 2016, 264, 78-87.

(26) Türke, M. T.; Bennati, M. Saturation factor of nitroxide radicals in liquid DNP by pulsed ELDOR experiments. Phys. Chem. Chem. Phys. 2011, 13, 3630 .

(27) Hwang, L.-P.; Freed, J. H. Dynamic effect of pair correlation function on spin relaxation by translational diffusion in liquids. J. Chem. Phys. 1975, 63, 4017-4025. 
(28) Polnaszek, C. F.; Bryant, R. G. Nitroxide radical induced solvent proton relaxation: Measurement of localized translational diffusion. J. Chem. Phys. 1984, 81, 4038-4045.

(29) Kruk, D.; Korpała, A.; Kowalewski, J.; Rössler, E. A.; Moscicki, J. ${ }^{1} \mathrm{H}$ relaxation dispersion in solutions of nitroxide radicals: Effects of hyperfine interactions with ${ }^{14} \mathrm{~N}$ and ${ }^{15} \mathrm{~N}$ nuclei. J. Chem. Phys. 2012, 137, 044512.

(30) Küçük, S. E.; Sezer, D. Multiscale computational modeling of 13 C DNP in liquids. Phys. Chem. Chem. Phys. 2016, 18, 9353-9357.

(31) Sezer, D.; Prandolini, M. J.; Prisner, T. F. Dynamic nuclear polarization coupling factors calculated from molecular dynamics simulations of a nitroxide radical in water. Phys. Chem. Chem. Phys. 2009, 11, 6626-6637.

(32) Neugebauer, P.; Krummenacker, J. G.; Denysenkov, V. P.; Helmling, C.; Luchinat, C.; Parigi, G.; Prisner, T. F. High-field liquid state NMR hyperpolarization: a combined DNP/NMRD approach. Phys. Chem. Chem. Phys. 2014, 16, 18781-18787.

(33) Türke, M. T.; Parigi, G.; Luchinat, C.; Bennati, M. Overhauser DNP with ${ }^{15} \mathrm{~N}$ labelled Frémy's salt at 0.35 T. Phys. Chem. Chem. Phys. 2012, 14, 502-510.

(34) Van Meerten, S. G.; Tayler, M. C.; Kentgens, A. P.; Van Bentum, P. J. Towards Overhauser DNP in supercritical $\mathrm{CO}_{2}$. J. Magn. Reson. 2016, 267, 30-36.

(35) Winkelmann, J. In Diffusion in Gases, Liquids and Electrolytes; Lechner, M. D., Ed.; Springer: Berlin, Heidelberg, 2017; Vol. 15B1, pp 317-319.

(36) Kruk, D.; Korpala, A.; Rössler, E.; Earle, K. A.; Medycki, W.; Moscicki, J. ${ }^{1} \mathrm{H}$ NMR relaxation in glycerol solutions of nitroxide radicals: Effects of translational and rotational dynamics. J. Chem. Phys. 2012, 136, 114504.

(37) O’Reilly, D. E.; Peterson, E. M. Self-diffusion coefficients and rotational correlation times in polar liquids. III. Toluene. J. Chem. Phys. 1972, 56, 2262-2266.

(38) Tironi, I. G.; Van Gunsteren, W. F. A molecular dynamics simulation study of chloroform. Mol. Phys. 1994, 83, 381-403.

(39) Müller-Warmuth, W.; van Steenwinkel, R.; Noack, F. Dynamic nuclear polarization experiments on ${ }^{19} \mathrm{~F}$ in solutions and their interpretation by the "Pulse model" of molecular collisions. $Z$. Naturforsch., A: Phys. Sci. 1968, 23, 506-513.

(40) Pronk, S.; Páll, S.; Schulz, R.; Larsson, P.; Bjelkmar, P.; Apostolov, R.; Shirts, M. R.; Smith, J. C.; Kasson, P. M.; van der Spoel, D.; Hess, B.; Lindahl, E. GROMACS 4.5: a high-throughput and highly parallel open source molecular simulation toolkit. Bioinformatics 2013, $29,845-854$.

(41) Stendardo, E.; Pedone, A.; Cimino, P.; Menziani, M. C.; Crescenzi, O.; Barone, V. Extension of the AMBER force-field for the study of large nitroxides in condensed phases: an ab initio parameterization. Phys. Chem. Chem. Phys. 2010, 12, 11697-11709. 